\title{
Hybrid Network For End-To-End Text-Independent Speaker Identification
}

\author{
Wajdi Ghezaiel*, Luc Brun ${ }^{\dagger}$ and Olivier LÉZORAY ${ }^{\dagger}$ \\ ${ }^{*}$ Normandie Université, UNICAEN, ENSICAEN, CNRS, NormaSTIC, Caen France \\ $\dagger$ Normandie Université, UNICAEN, ENSICAEN CNRS, GREYC Caen, France \\ Email:wajdi.ghezaiel@ensicaen.fr, luc.brun@ensicaen.fr, olivier.lezoray@unicaen.fr
}

\begin{abstract}
Deep learning has recently improved the performance of Speaker Identification (SI) systems. Promising results have been obtained with Convolutional Neural Networks (CNNs). This success is mostly driven by the advent of large datasets. However in the context of decentralized commercial applications, collection of large amount of training data is not always possible. In addition, robustness of a SI system is adversely effected by short utterances. Therefore, in this paper, we propose a novel textindependent speaker identification system able to identify speakers by learning from only few training short utterances examples. To achieve this, we combine a two-layer wavelet scattering network coupled with a CNN. The proposed architecture takes variable length speech segments. To evaluate the effectiveness of the proposed approach, Timit and Librispeech datasets are used in the experiments. Our experiments shows that our hybrid architecture provides satisfactory results under the constraints of short and limited number of utterances. These experiments also show that our hybrid architecture are competitive with the state of the art.
\end{abstract}

\section{INTRODUCTION}

Speaker identification (SI) is an important biometric recognition technology. It is the task of identifying a person, based on a given speech signal and enrolled speaker records [1]. SI has gained great popularity in a wide range of applications, such as access user control, transaction authentication, forensics and personalization. After decades of research, significant performance improvement has been gained and some SI systems have been deployed in some practical applications [2], [3], [4]. In spite of these great achievements, current SI systems perform well only if the enrollment and test utterances are well matched, otherwise the performance will be seriously degraded. Moreover, many applications require very good accuracy even with short duration utterances. However, the performance of SI systems degrade with short utterances of about 5-10 seconds [5]. Different studies [6], [7] [8] have shown that the use of short segments may induce a drastic drop of the performances of authentication systems. This drop in performance is mainly due to the low amount of information on each speaker that is usually extracted from such short sequences. Speaker identification with only few and short utterances is thus a challenging problem.

Most of traditional SI systems are based on features relying on speech production and perception, such as Mel-Frequency Cepstral Coefficients (MFCCs), and on unsupervised generative models. During the training phase, MFCC features are used to train a Gaussian Mixture model (GMM) and to build an Universal Background Model (UBM) [9]. The GMM-UBM framework represents the speaker and channel independent attributes over their Gaussian components. However, it has been shown [10], [11] that it is beneficial to further process this vector by extracting intermediate vectors called i-vectors. During the authentication phase, an i-vector is extracted from a given speech sample and is compared to the reference $\mathrm{i}$-vector, either with a simple cosine distance or with more complex techniques such as Probabilistic Linear Discriminant Analysis (PLDA) [12]. However, performance of these baseline methods suffer of sensitivity to lexical variability for short utterances [13].

Recently, deep learning has appeared in many pattern recognition fields. It has shown remarkable success in many fields such as image recognition [14] and natural language processing [15]. In speaker identification, a similar trend has been observed. Deep Neural Networks (DNNs) have been used with the i-vector framework to compute Baum-Welch statistics [16], or for frame-level feature extraction [17]. DNNs have also been proposed for direct discriminative speaker classification, as witnessed by the recent literature on this topic [18], [19]. Lately, there was an increasing number of studies trying the use of convolutional neural network [20] in numerous speech tasks [21], [22]. Some works have proposed to directly feed networks with spectrogram bins [23], [24] or even with raw waveforms [25], [26]. Among DNNs, CNNs have the most suitable architecture for processing raw speech samples, since weight sharing, local filters, and pooling constitute precious tools to discover robust and invariant representations. However, CNNs networks require numerous labeled training examples along with considerable computational resources and time to achieve effective learning. In a decentralized setting where only few labeled data with short duration are available, the training becomes difficult and requires a lot of regularization.

Recently, Mallat et al. [27] have proposed Scattering wavelet networks as a class of Convolutional Neural Networks (CNNs) with fixed weights. They have largely investigated the wavelet scattering transform (WST) framework and its properties. WST possesses the same properties used in CNN to extract reliable features from data. Additionally, the WST can extract reliable information at different scaling levels of decomposition. Also, it has been proved that the wavelet scattering coefficients are more informative than a Fourier transform 
when dealing with short variation signals or small deformation and rotation invariant [28], [29].

Scattering representations can be plugged into any classification or regression system, be it shallow or deep. The WST was tested on handwriting image data to extract the features where it achieved good performance [28]. WST has enjoyed significant success in various audio [27] and biomedical [30] signal classification tasks. WST demonstrated promising results on the TIMIT dataset for phonetic classification [31] and recognition [32].

In this paper, we propose a two-stage feature extraction framework using a two-layer wavelet scattering network coupled with a CNN for SI system. We explore the use of the WST for feature extraction along with a convolutional neural network. In this hybrid deep learning network, the use of a two-stage feature extraction framework can be helpful when there is a lack of data. This provides features of the same signal at different scales and captures its dominant energy. Such advantages could be useful when dealing with short duration utterances. The proposed network takes variable length speech segments. It is trained at the frame-level using the extracted features. The system has been evaluated on both Timit and Librispeech datasets and it has achieved better results than the state-of-the-art.

The remainder of this paper is organized as follows. Section II presents the wavelet scattering transform. Section III describes the proposed hybrid architecture, which is composed in a cascade of a scattering transform and a convolutional neural network. Section IV discusses the experimental setup and the corresponding results obtained by the proposed system as well as the ones provided by related systems.

\section{WAVELET SCATTERING TRANSFORM}

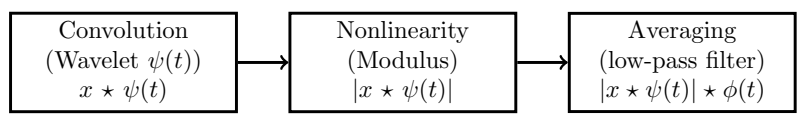

Fig. 1. Wavelet scattering transform processes, where $x$ is the input data, $\psi$ a wavelet function and $\phi$ an averaging low-pass filter.

To produce a wavelet scattering transform [27] of an input signal $x$, three successive operations are required: convolution, nonlinearity, and averaging as described in Figure 1. The scattering transform coefficients are obtained with the averaging of wavelet modulus coefficients by a low-pass filter $\phi$. Let a wavelet $\psi(t)$ be a band pass filter with a central frequency normalized to 1 , and $\psi_{\lambda}(t)$ a wavelet filter bank, which is constructed by dilating the wavelet:

$$
\psi_{\lambda}(t)=\lambda \psi(\lambda t)
$$

where $\lambda=2^{\frac{j}{Q}}, \forall j \in \mathbb{Z}$ and $Q$ is the number of wavelets per octave.

The bandwidth of the wavelet $\psi(t)$ is of the order $\frac{1}{Q}$, and as a result, the filter bank is composed of band pass filters

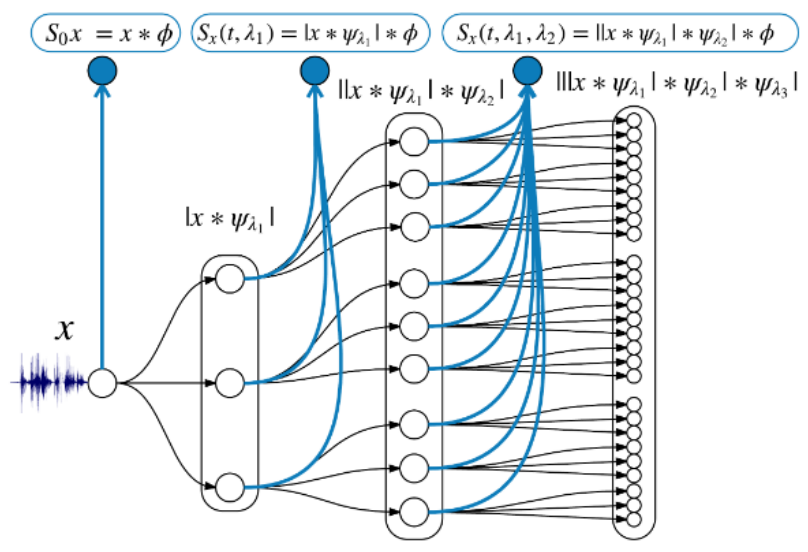

Fig. 2. Hierarchical representation of scattering coefficients at multiple layers [27].

which are centered in the frequency domain in $\lambda$ and have a frequency bandwidth $\frac{\lambda}{Q}$.

At the zero order, we have a single coefficient given by $S_{0} x(t)=x \star \phi(t)$, which is close to zero for audio signals. At the first order, we set $Q_{1}=8$ for speech signals, which defines wavelets having the same frequency resolution as mel-frequency filters. Approximate mel-frequency spectral coefficients are obtained by averaging the wavelet modulus coefficients with $\phi$ :

$$
S_{1} x\left(t, \lambda_{1}\right)=\left|x \star \psi_{\lambda_{1}}\right| \star \phi(t)
$$

The second order coefficients capture the high-frequency amplitude modulations occurring at each frequency band of the first layer and are obtained by:

$$
S_{2} x\left(t, \lambda_{1}, \lambda_{2}\right)=|| x \star \psi_{\lambda_{1}}\left|\star \psi_{\lambda_{2}}\right| \star \phi(t)
$$

The wavelets $\psi_{\lambda_{2}}$ have an octave resolution $Q_{2}$ which may be different from $Q_{1}$. We set $Q_{2}=1$ for speech signals, to defines wavelets with more narrow time support, which are better adapted to characterize transients and attacks. We get a sparse representation which means concentrating the signal information over as few wavelet coefficients as possible. These coefficients are averaged by the low pass filter $\phi$, which ensures local invariance to time-shifts, as with the first-order coefficients.

Figure 2 shows the hierarchy of scattering coefficients. This somewhat resembles to the structure of deep neural networks, although that in the scattering transform, each layer provides some output, while the only output of most of deep neural networks is provided by the last layer. This decomposition on first and second orders scattering coefficients is applied to the time domain signals. Second order features are normalized by first order features, to ensure that the higher order of scattering depends on the amplitude modulation component of the speech signal. The first and second orders of the scattering transform are concatenated to form a scattering feature vector for a 


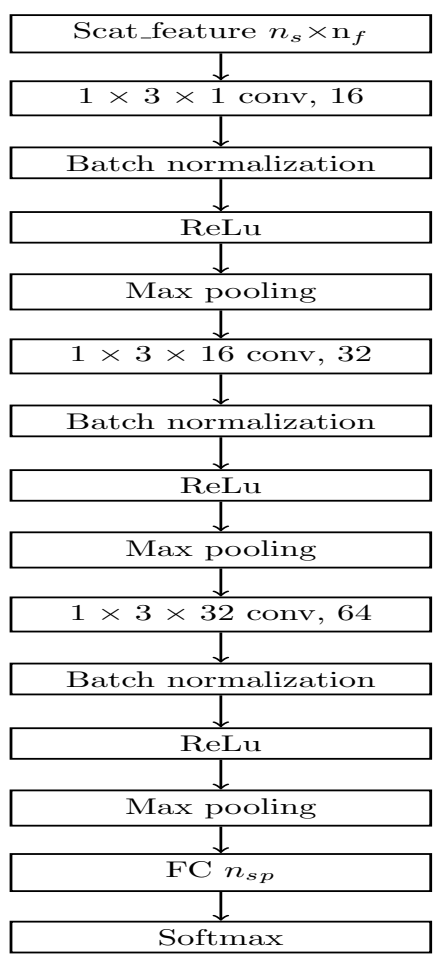

Fig. 3. The proposed Hybrid Wavelet Scattering Transform Convolutional Neural Network (HWSTCNN) architecture.

given frame. The scattering features include log-mel features as well as higher order features to preserve the greatest details in the speech signal [27]. This representation is invariant to time shifts and is stable to deformations. Hence, to ensure invariability to frequency translation on a logarithmic scale like translation of speaker formants, a scattering transform is performed along log-frequency. The logarithm is applied to each coefficients of the scattering feature vector. It is thus locally translation invariant in time and $\log$ frequency, and stable to time and frequency deformations.

\section{Hybrid Network ARChitecture}

An ideal model for SI system should take variable length speech segments and produce a discriminating output descriptor. The distance between descriptors of different speakers must be larger than those of the same speaker. To satisfy all mentioned properties, Figure 3 shows the proposed Hybrid Wavelet Scattering Transform Convolutional Neural Network (HWSTCNN). The network consists of two parts: feature extraction and frame level embedding. The scattering network is coupled with convolutional layers to extract frame level features, and dense classification layer to construct speaker frame embedding.

The network is shown in Figure 3 and described in more details in the following paragraphs. The proposed architecture consists in two scattering network layers, namely, ScatLayer, three 2D convolutional layers, and one fully connected layer. Scat-Layer performs scattering wavelet transform on
TABLE I

HWSTCNN ARCHITECTURE. EACH ROW SPECIFIES THE \# OF CONVOLUTIONAL FILTERS, THEIR SIZES, AND THE \# FILTERS.

\begin{tabular}{|c|c|c|}
\hline Layer name & Hybrid model & Output \\
\hline Input & -- & $n \times 1$ \\
\hline ScatNet layer & -- & $n_{s} \times n_{f} \times 1$ \\
\hline Conv1 block & $\begin{array}{l}\text { conv2D, } 1 \times 3 \times 1,16 \\
\text { bn } \\
\text { relu }\end{array}$ & $n_{s} \times n_{f} \times 16$ \\
\hline Pooling & $\begin{array}{l}\operatorname{maxpool}, 1 \times 2, \quad \text { stride } \\
(2,1)\end{array}$ & $n_{s} \times n_{f} / 2 \times 16$ \\
\hline Conv2 block & $\begin{array}{l}\text { conv2D, } 1 \times 3 \times 16,32 \\
\text { bn } \\
\text { relu }\end{array}$ & $n_{s} \times n_{f} / 2 \times 32$ \\
\hline Pooling & $\begin{array}{l}\operatorname{maxpool}, 1 \times 2, \text { stride } \\
(2,1)\end{array}$ & $n_{s} \times n_{f} / 4 \times 32$ \\
\hline Conv3 block & $\begin{array}{l}\text { conv2D, } 1 \times 3 \times 32,64 \\
\text { bn } \\
\text { relu }\end{array}$ & $n_{s} \times n_{f} / 4 \times 64$ \\
\hline Pooling & $\begin{array}{l}\text { maxpool, } 1 \times 2, \text { stride } \\
(2,1)\end{array}$ & $n_{s} \times n_{f} / 8 \times 64$ \\
\hline Embedding & fc, $n_{s p}$ & $1 \times 1 \times n_{s p}$ \\
\hline Loss & softmax & -- \\
\hline
\end{tabular}

overlapping frames $(500 \mathrm{~ms}$ with $125 \mathrm{~ms}$ skip rate) in timedomain signal. After the Scat-Layer, three convolutional layers are followed by one fully connected layer. Standard CNN pipeline (pooling, batch normalization, ReLU activation) was employed. Final softmax layer performs speaker classification.

ScatNet layer is composed of two scattering wavelet transform layers. The first layer contains 8 Gabor wavelets per octave and the second has 1 Morlet wavelet per octave. This configuration was chosen to match the frequency resolution of Mel filters at the first level. The second order of the scattering transform recovers the lost information. Averaging window length was set to $32 \mathrm{~ms}$. Later, coefficients are normalized and log-transformed. Therefore, the representation of speech signal using the first and the second orders of the scattering transform extends the MFCC representation and doesn't loose information. These scattering coefficients are computed using a publicly available toolbox [27].

Each convolutional layer is formed by a 2D filter of length 3 and batch normalization. They are followed by a max-pooling layer, with pooling size $1 \times 2$ and stride $1 \times 2$. The number of filters is respectively 16,32 and 64 . A fully connected layer with $n_{s p}$ hidden neurons, where $n_{s p}$ is the number of speakers to be identified, is connected to categoral softmax layer. The softmax produces a probability distribution per frame over the target speakers in the dataset.

We use rectified linear units as activation functions in all layers. Stochastic gradient descent was used as an optimizer with a learning rate of 0.001 and 0.9 momentum. The network is trained with mini batches of size 64 for 10 epochs. The proposed architecture is shown in Figure 3 and details such as the number of filters and kernel sizes are summarized in Table I. This architecture takes raw speech frames with timewindows of $500 \mathrm{~ms}$ and with a skip rate of $125 \mathrm{~ms}$ to produce speaker embedding at frame-level. The amount of parameters in this neural network is 18,1 millions which is less than the 
actual state-of-the-art, as it will be shown in the next sections. Coupling a scattering network with a convolutional network for building our hybrid architecture can reduce the instabilities in the first layers as the wavelet scattering transform is stable and non-expansive. By reducing the variability at feature extraction stage, the proposed hybrid architecture can generate discriminative feature information at frame level. This hybrid architecture has the capability to reduce the required depth and spatial dimension of the deep learning networks, which makes the strength of using both scattering transform and CNN.

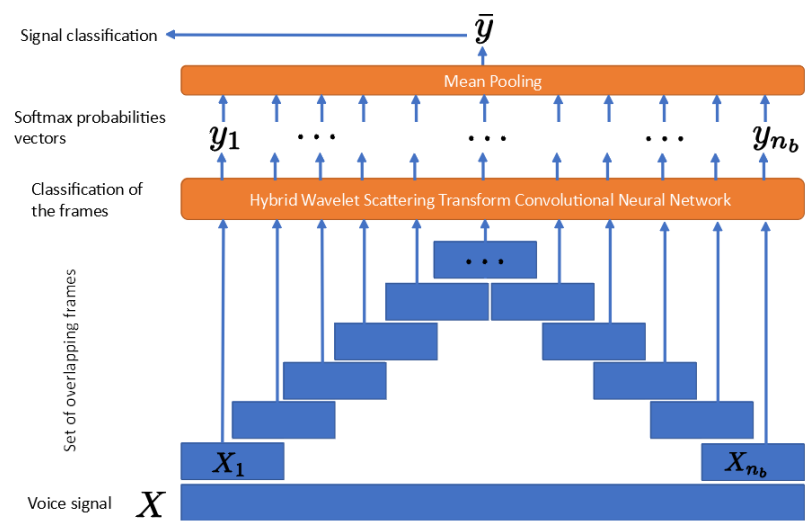

Fig. 4. Hybrid Wavelet Scattering Transform Convolutional Neural Network.

In the testing phase of our system, a speech utterance $X$ to be classified is divided into $n_{b}$ overlapping frames of length $n_{f}$. Each frame shares the first part with the previous frame and the last part with the next frame. Each frame $X_{i}$ is fed to our HWSTCNN to predict the frame speaker label. Softmax probabilities $y_{i}^{k}=\operatorname{Pr}\left(s_{k} \mid X_{i}\right)$ are calculated to estimate if the frame $X_{i}$ is from the speaker $s_{k}$ among the $n_{s p}$ speakers. These speaker membership probabilities $y_{i} \in \mathbb{R}^{n_{s p}}$ constitute a vector for each frame $i$. Finally, the mean membership probability vector $\bar{y}$ of the whole utterances is given by the mean of all the stored probability vectors computed per frame. The estimated speaker label for the whole speech utterance $X$ corresponds to the speaker of maximum of probability: label $=\arg \max _{j \in\left[1, n_{s p}\right]} \bar{y}^{j}$ with $\bar{y}^{j}$ the $j^{\text {th }}$ column of the $\bar{y}$ vector and $n_{s p}$ the total number of speakers.

Figure 4 describes the process of labeling speech utterances. Speech frames are first fed into our HWSTCNN to predict frame speaker probability vectors. These frame-level predictions are then aggregated into a whole-utterance-level prediction using the mean of the obtained softmax frame-level probabilities. Therefore the speaker's probabilities prediction is initially made per frame and thereafter is converted into a final single speaker vector probability for the whole utterance. Finally, the estimated speaker label corresponds to the one that has the highest probability among the ones of the average vector probability $\bar{y}$.
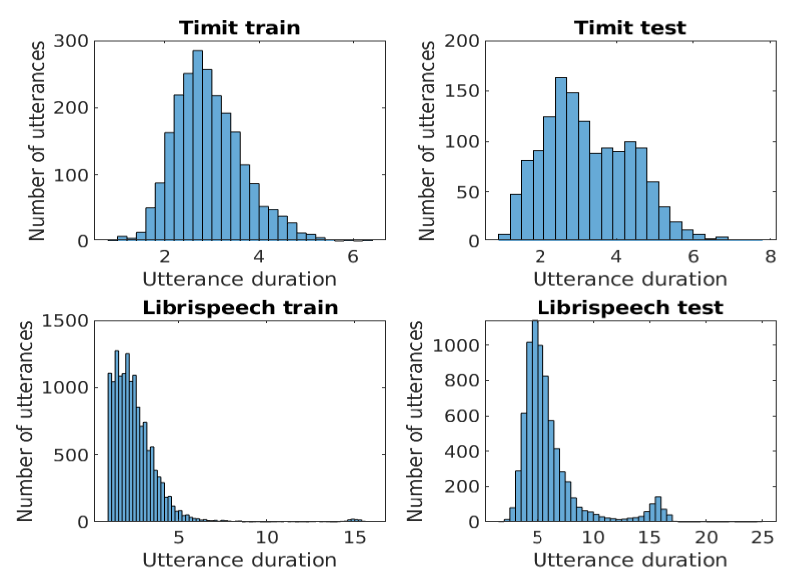

Fig. 5. Distribution of the utterance lengths in Timit and Librispeech databases.

\section{EXPERIMENTS}

This section describes the experiments and the results obtained with our approach and related systems.

\section{A. Dataset and experimental setting}

Two datasets are used in the experiments, TIMIT [33] and LibriSpeech [34].

- The TIMIT dataset contains studio quality recordings of 630 speakers (192 female, 438 male), sampled at $16 \mathrm{kHz}$, covering the eight major dialects of American English. Each speaker reads ten phonetically rich sentences. We consider only 462 speakers from TIMIT. We use only 8 sentences for each speaker, the "SX" (5 sentences) and the "SI" (3 sentences). These 8 sentences are different from one another, and different across speakers. The "SX" sentences have an average duration of 3.2 seconds while "SI" sentences have an average duration of 2.9 seconds. The "SX" sentences are used to train the system, while the "SI" sentences are used to test. The TIMIT dataset is considered as a challenging task for end to end systems due to its very limited amount of available training data (less than 5 hours).

- The LibriSpeech database consists in audio books readout-loud by 2484 speakers, 1283 male and 1201 female volunteers who recorded their voices spontaneously. The speech signal is usually clean, but the recording device and channel conditions vary a lot between different utterances and speakers. We decided to keep 7 utterances of each speaker for training, and 3 utterances as a fixed test set for evaluation.

In Timit and Librispeech datasets, the total duration of training sentences is about 12-15 seconds for each speaker and test sentences duration is about of 2-5 seconds. Figure 5 shows the distribution of the utterances durations. The average duration was of $4 \mathrm{~s}$, the minimum was of $1 \mathrm{~s}$, and the maximum was of $17 \mathrm{~s}$. Utterances with durations of less than 4 seconds represented about $87 \%$ of the data. 
TABLE II

PARTITION OF UTTERANCES FOR THE SPEAKER IDENTIFICATION TASK.

\begin{tabular}{|c|c|c|c|c|}
\cline { 2 - 5 } \multicolumn{1}{c|}{} & Train & Test & Total number & Total Duration \\
\hline Timit & 2310 & 1386 & 3696 & $3 \mathrm{~h} 15 \mathrm{mn}$ \\
\hline Librispeech & 17388 & 7452 & 22356 & $22 \mathrm{~h} 45 \mathrm{mn}$ \\
\hline
\end{tabular}

Table II presents utterances partition for training and testing for both datasets. This partition has been also used in original implantation of SincNet [35].

To validate the effectiveness of our model, we built $8 \mathrm{kHz}$ and $16 \mathrm{kHz}$ versions of our system. Timit and Librispeech datasets are downsampled to $8 \mathrm{kHz}$. Finally, we use 3 -fold cross-validation to evaluate the performance of the proposed system on Librispeech dataset.

Experiments are conducted on the full and short length conditions. We do not apply any pre-processing to the raw waveforms, such as pre-emphasis, silence removal, detection and removal of unvoiced speech. Hence, non speech intervals at the beginning and end of each sentence are conserved. Scattering transform is computed up to depth 2 with speech frames of $500 \mathrm{~ms}$. The first layer contains 8 Gabor wavelets per octave while the second one has 1 Morlet wavelet per octave. The size of the averaging window is set to $32 \mathrm{~ms}$. Later, coefficients are normalized and log-transformed. Stochastic gradient descent is used as an optimizer with a learning rate of 0.001 and 0.9 momentum. The network is trained with mini batches of size 64 for 10 epochs. Our implementation is based on Scatnet [27] and deep learning Matlab toolboxes.

\section{B. Related systems}

In order to evaluate the performance of our proposed system, two alternative state-of-the-art systems were investigated: SincNet [35] and CNN-Raw [36] systems for speaker identification.

- SincNet is a novel end-to-end neural network architecture, that directly receives raw waveforms as input. The first 1D convolutional layer of SincNet is composed by Sinc functions. SincNet convolves the waveform with a set of parametric sinc functions that implement band-pass filters. The filters are initialized using the Mel-frequency filter bank and their low and high cutoff frequencies are adapted with standard back-propagation as any other layer. The first layer performs Sinc based convolutions, using 80 filters of length 251 . The remaining two layers use 60 filters of length 5 . Next, three fully-connected layers composed of 2048 neurons and normalized with batch normalization are applied. All hidden layers use leaky-ReLU non-linearity. Frame-level binary classification is performed by applying a softmax classifier and cross-entropy criteria [35].

- In the CNN-Raw system, the raw waveform is fed directly to the first layer. Three convolution layers are used to perform the feature mapping. Each convolution layer is composed of 80 filters followed by a max pooling. Next, three fully-connected layers composed of 2048 neurons and normalized with batch normalization are applied. All hidden layers use leaky-ReLU non-linearities. Framelevel binary classification is performed by applying a softmax classifier and cross-entropy criteria [36].

SincNet and CNN-raw systems perform silence detection technique to remove non-speech intervals at the beginning and end of each sentence. Sentences with internal silences lasting more than $125 \mathrm{~ms}$ were split into multiple chunks. Both networks are trained with 2900 epochs and batches of size 128 on Librispeech dataset. The number of parameters in CNN-raw is about 27.6 millions while the one of SincNet is about 26.5 millions. Table III summarizes the number of

TABLE III

NUMBER OF PARAMETERS AND EPOCHS FOR OUR SYSTEM AND RELATED SYSTEMS.

\begin{tabular}{|c|c|c|c|}
\cline { 2 - 4 } \multicolumn{1}{c|}{} & SincNet & CNN & HWSTCNN \\
\hline Parameters $\times \mathbf{1 0}^{\mathbf{6}}$ & 26,5 & 27,6 & $\mathbf{1 8 , 1}$ \\
\hline
\end{tabular}

learning parameters of all tested methods. We observe that the number of learning parameters required by our method is lower than the ones of SincNet and CNN-Raw by about $33 \%$.

\section{TABLE IV}

IDENTIFICATION ACCURACY RATE (\%) OF THE PROPOSED HWSTCNN ON $8 \mathrm{~K}$ AND $16 \mathrm{~K}$ DATA TRAINED AND TESTED WITH FULL UTTERANCES.

\begin{tabular}{|c|c|c|}
\cline { 2 - 3 } \multicolumn{1}{c|}{} & $\mathbf{8 k}$ & $\mathbf{1 6 k}$ \\
\hline LibriSpeech & 97.38 & $\mathbf{9 9 . 2 8}$ \\
\hline TIMIT & 85.93 & $\mathbf{9 8 . 1 2}$ \\
\hline
\end{tabular}

\section{Results}

In order to evaluate our proposed speaker identification system we use the identification accuracy rate which is equal to the number of correct identifications over the number of speakers to test. In Table IV, we report the effect of sampling frequency on system performance. As expected, results show that our system performs better on $16 \mathrm{kHz}$ than $8 \mathrm{kHz}$ data. However, let us note that the correct identification rates decrease by only $2 \%$ between $16 \mathrm{KHz}$ and $8 \mathrm{KHz}$ data. Our system remains thus competitive for low sampling frequency rate. The table shows that the effect of coupling the first convolution layer of CNN with WST, improves the identification performance.

Correct identification rates for different methods are shown in Table V. Results are compared on both TIMIT and Librispeech datasets. Results from this table shows that our hybrid network obtains significant robustness on both datasets. Our system outperforms both SincNet and CNN-Raw systems under Librispeech dataset. It achieves a relative improvement of about $0.37 \%$ over CNN-raw and $0.35 \%$ over SincNet. For the TIMIT dataset, our system achieves $98.12 \%$ accuracy. However, both SincNet and CNN-Raw outperform our proposed system. Both these approaches of Sincnet and CNN-raw use signal pre-processing techniques, they use silence removal 
technique to detect speech segments. In our case, no preprocesing is performed and the original voice signal is directly fed to the system. Evaluation of our proposed system with the same pre-processing on database TIMIT gives $97.81 \%$. This decrease is due to the limited amount of data in training and testing. Indeed, the processed TIMIT dataset has a total duration of only $2 \mathrm{~h} 35 \mathrm{mn}$.

TABLE V

IDENTIFICATION ACCURACY RATE (\%) OF THE PROPOSED HWSTCNN AND RELATED SYSTEMS TRAINED AND TESTED WITH FULL UTTERANCES.

\begin{tabular}{|c|c|c|}
\cline { 2 - 3 } \multicolumn{1}{c|}{} & LibriSpeech & TIMIT \\
\hline CNN-raw & 98.91 & 98.62 \\
\hline SincNet-raw & 98.93 & $\mathbf{9 9 . 1 3}$ \\
\hline HWSTCNN & $\mathbf{9 9 . 2 8}$ & 98.12 \\
\hline
\end{tabular}

TABLE VI

IDENTIFICATION ACCURACY RATE (\%) OF THE PROPOSED HWSTCNN AND RELATED SYSTEMS TRAINED WITH EQUAL PARAMETERS ON LIBRISPEECH.

\begin{tabular}{|c|c|c|c|}
\cline { 2 - 4 } \multicolumn{1}{c|}{} & SincNet-raw & CNN-raw & HWSTCNN \\
\hline full-full & 98.93 & 98.91 & $\mathbf{9 9 . 4 6}$ \\
\hline
\end{tabular}

We further investigate the performances of our system in Table VI. We use the same deep CNN architecture used in Sincnet. This architecture is much deeper than the one we have considered and this augments the number of parameters, as seen in Table III. An increase of $0.17 \%$ is proved in accuracy performance. This experiment shows however the benefit of using the scattering transform, as better results are obtained by replacing the SincNet filters with the scattering transform. HWSTCNN converges faster after about 10 epochs of training and achieves better end task performance. Whereas SincNet and CNN-raw converge after about 1500 epochs of training.

TABLE VII

IDENTIFICATION ACCURACY RATE (\%) OF THE PROPOSED HWSTCNN ON LIBRISPEECH DATASET TRAINED AND TESTED WITH DIFFERENT UTTERANCES DURATIONS

\begin{tabular}{|c|c|c|c|}
\hline \multirow{2}{*}{ Test } & \multicolumn{3}{|c|}{ Train utterance duration } \\
\cline { 2 - 4 } & $\mathbf{8 s}$ & $\mathbf{1 2 s}$ & full \\
\hline $\mathbf{1 . 5 s}$ & 96.86 & 97.20 & 97.38 \\
\hline $\mathbf{3 s}$ & 98.76 & 98.93 & 98.97 \\
\hline full & 99.12 & 99.25 & 99.28 \\
\hline
\end{tabular}

We report in Table VII the effect of training utterances duration per speaker on performances. We split the training data to obtain a total duration of $8 \mathrm{~s}$ or $12 \mathrm{~s}$ per speaker. Full train duration is about $14 \mathrm{~s}$. This table depicts the correct identification rates for $1.5 \mathrm{~s}, 3 \mathrm{~s}$ and full duration of testing utterances. We observe that the proposed methods obtains significant robustness, which indicates that the proposed method is able to extract speaker identity features in different training and testing conditions. Our system gives higher accuracy rate for all conditions of short-utterance task. As shown in Table VII, varying the number of samples per speaker and thus the total duration for training induces a variation of only $0.15 \%$ of the accuracy. On the other hand, using $3 \mathrm{~s}$ duration instead of $1.5 \mathrm{~s}$ induces an small increase of the accuracy of about $0.5 \%$. Our system is thus able to construct discriminating speakers models with few number of training data but provides better results with test samples of at least $3 \mathrm{~s}$.

TABLE VIII

IDENTIFICATION ACCURACY RATE (\%) OF THE PROPOSED HWSTCNN AND RELATED SYSTEMS TRAINED ON LIBRISPEECH DATASET AND TESTED WITH DIFFERENT UTTERANCES DURATIONS.

\begin{tabular}{|c|c|c|c|}
\cline { 2 - 4 } \multicolumn{1}{c|}{} & SincNet-raw & CNN-raw & HWSTCNN \\
\hline 1.5s-full & 91.51 & 94.28 & $\mathbf{9 7 . 3 8}$ \\
\hline 3s-full & 97.57 & 96.87 & $\mathbf{9 8 . 9 7}$ \\
\hline
\end{tabular}

TABLE IX

IDENTIFICATION ACCURACY RATE (\%) OF THE PROPOSED HWSTCNN AND RELATED SYSTEMS TRAINED ON TIMIT DATASET AND TESTED WITH DIFFERENT UTTERANCES DURATIONS.

\begin{tabular}{|c|c|c|c|}
\cline { 2 - 4 } \multicolumn{1}{c|}{} & SincNet-raw & CNN-raw & HWSTCNN \\
\hline 1.5s-full & $\mathbf{9 7 . 4 0}$ & 80.00 & 91.41 \\
\hline 3s-full & $\mathbf{9 8 . 7 0}$ & 97.47 & 97.76 \\
\hline
\end{tabular}

Tables VIII and IX show accuracy results for Librispeech and TIMIT datasets with different short utterance durations. It reveals that the proposed HWSTCNN gets the highest average accuracy on the Librispeech dataset. With a speech duration of $3 \mathrm{~s}$, HWSTCNN yields $2.1 \%$ and $1.4 \%$ of relative improvement over CNN-raw and SincNet respectively. Moreover, HWSTCNN accuracy with speech duration of $1.5 \mathrm{~s}$ yields $3.1 \%$ and $5.87 \%$ of relative improvement over CNNraw and SincNet respectively. For the TIMIT dataset, table IX shows that our proposed system outperforms CNN-raw with both speech duration. However for this dataset our results are less good than SincNet. We believe that adding more layers to our model could further improve our results.

TABLE X

IDENTIFICATION ACCURACY RATE (\%) OF THE PROPOSED HWSTCNN AND RELATED SYSTEMS TRAINED AND TESTED ON LIBRISPEECH DATASET WITH 3-FOLD CROSS-VALIDATION.

\begin{tabular}{|c|c|c|c|c|c|}
\cline { 2 - 6 } \multicolumn{1}{c|}{} & Fold1 & Fold2 & Fold3 & Average & Std Dev \\
\hline CNN-raw & 98.71 & 98.67 & 98.88 & 98.75 & 0.11 \\
\hline SincNet-raw & 98.73 & 98.63 & 98.86 & 98.74 & 0.12 \\
\hline HWSTCNN & $\mathbf{9 8 . 7 8}$ & $\mathbf{9 8 . 7 1}$ & $\mathbf{9 8 . 8 9}$ & $\mathbf{9 8 . 7 9}$ & $\mathbf{0 . 0 9}$ \\
\hline
\end{tabular}

For method validation, 3-fold cross-validation is performed to verify the accuracy and generalization capabilities of our proposed HWSTCNN. Table X shows accuracy of our proposed method and related methods under 3 -fold cross validation. The mean accuracy for our model HWSTCNN using 3 -fold cross-validation is $98.79 \%$. The table highlights that HWSTCNN outperforms the other models, showing a relative improvement of about $0.4 \%$ over SincNet and CNN-raw. To assess the statistical significance between the obtained results of the compared methods, a significance level of 0.05 was used, that is, when the p-value is less than 0.05, the performance difference of two methods is statistically significant. 
HWSTCNN achieved a statistically significance of $3.34 \%$ and 7.36\% respectively with SincNet and CNN-raw.

\section{CONCLUSION}

In this paper, we have proposed HWSTCNN a speaker identification system that learns speaker discriminating information directly from raw speech signals using scattering wavelet transform and CNNs. We have explored the potential advantage of WST in extracting robust speaker representation. We have demonstrated that by coupling CNN with scattering wavelet network, we are able to compute a stable description of speaker identity information. Experimental results on TIMIT and Librispeech corpuses have shown that the proposed system can achieve dominant results in clean condition with limited amount of data. We have shown the effectiveness of our hybrid architecture for speaker identification with different utterances duration used in training and testing phases. Our results show that our hybrid model is competitive with SincNet and CNN-raw methods on the same databases. Beyond extensively experiments and performance improvements, combining WST and CNN demonstrates the efficacy of the scattering wavelet layer in learning merged feature and enabling a better and lossless latent representation of the speech signal. These results show significant promise for considerable improvement in speaker identification and in speaker verification which we plan to study in future works. In future work, we would like to evaluate HWSTCNN on other popular speaker recognition tasks, such as speaker verification and explore other dataset such as VoxCeleb. Inspired by the promising results obtained in this paper, this work could be extended in future works to other tasks, such as emotion recognition, speech separation, and music processing.

\section{ACKNOWLEDGMENTS}

This work was supported by BPI France, project HomeKeeper.

\section{REFERENCES}

[1] H. Beigi, "Fundamentals of Speaker Recognition," Springer US, 2011.

[2] D. A. Reynolds, "An overview of automatic speaker recognition technology," in Acoustics, speech, and signal processing (ICASSP), 2002 IEEE international conference on, vol. 4. IEEE, 2002, pp. IV-4072.

[3] T. Kinnunen and H. Li, "An overview of text-independent speaker recognition: From features to supervectors," Speech communication, vol. 52, no. 1 , pp. 12-40, 2010.

[4] J. H. Hansen and T. Hasan, "Speaker recognition by machines and humans: A tutorial review," IEEE Signal processing magazine, vol. 32, no. 6, pp. 74-99, 2015.

[5] P. Kenny, T. Stafylakis, P. Ouellet, M. J. Alam, and P. Dumouchel, "Plda for speaker verification with utterances of arbitrary duration," in 2013 IEEE International Conference on Acoustics, Speech and Signal Processing, May 2013, pp. 7649-7653.

[6] A. Poddar, M. Sahidullah, and G. Saha, "Speaker verification with short utterances: a review of challenges, trends and opportunities," IET Biometrics, vol. 7, no. 3, pp. 91-101, 2018.

[7] Rohan Kumar Das and S. R. M. Prasanna, "Speaker verification for variable duration segments and the effect of session variability," Lecture Notes in Electrical Engineering, pp. 193-200, 2015.

[8] A. Kanagasundaram, R. Vogt, D. Dean, and S. Sridharan, "i-vector based speaker recognition on short utterances," in Proc. of Interspeech , 2011.
[9] D. Reynolds, T. Quatieri, and R. Dunn, "Speaker verification using adapted gaussian mixture models," Digital Signal Processing, vol. 10, no. 1, pp. 19-41, 2000.

[10] N. Dehak, P. J. Kenny, R. Dehak, P. Dumouchel, and P. Ouel- let, "Frontend factor analysis for speaker verification," IEEE Transactions on Audio, Speech, and Language Processing, vol. 19, no. 4, pp. 788-798, 2011.

[11] Wei Li, Tianfan Fu, and Jie Zhu, "An improved i-vector ex- traction for speaker verification," EURASIP Journal on Audio, Speech and Music Processing , pp. 1-9, 2015.

[12] S. J. Prince and J. H. Elder, "Probabilistic linear discriminant analysis for inferences about identity," in Proc. of International Conference on Computer Vision , 2007.

[13] W. Li, T. Fu, H. You, J. Zhu, and N. Chen, "Feature sparsity analysis for i-vector based speaker verification," Speech Communication, vol. 80, pp. 60-70, 2016.

[14] A. Krizhevsky, I. Sutskever, and G. E. Hinton, "Imagenet classification with deep convolutional neural networks," in Proc. of Advances in Neural Information Processing Systems , 2012.

[15] R. Collobert and J. Weston, "A unified architecture for natural language processing: Deep neural networks with multitask learning," in Proc. of the International Conference on Machine Learning , 2008.

[16] P. Kenny, V. Gupta, T. Stafylakis, P. Ouellet, and J. Alam, "Deep neural networks for extracting baum-welch statistics for speaker recognition," in Proc. of Speaker Odyssey, 2014.

[17] S. Yaman, J. W. Pelecanos, and R. Sarikaya, "Bottleneck features for speaker recognition," in Proc. of Speaker Odyssey, 2012, pp. 105-108.

[18] E. Variani, X. Lei, E. McDermott, I. L. Moreno, and J. GonzalezDominguez, "Deep neural networks for small foot-print text-dependent speaker verification," in Proc. Of ICASSP , 2014, pp. 4052-4056

[19] D. Snyder, D. Garcia-Romero, G. Sell, D. Povey, and S. Khudanpur, "X-vectors: Robust dnn embeddings for speaker recognition," in Proc. of ICASSP , 2018.

[20] Yann LeCun and Yoshua Bengio, "Convolutional networks for images, speech, and time series," in The hand-book of brain theory and neural networks , 1995, vol. 3361, p. 1995.

[21] Abdel-Hamid O., Mohamed Abdel-rahman, Jiang Hui, Deng Li, Penn Gerald, and Dong Yu, "Convolutional neural networks for speech recognition," IEEE Transactions on Audio, Signal, and Language Processing , vol. 22, no. 10, pp. 1533-1545, 2014.

[22] T. N. Sainath, A. Mohamed, B. Kingsbury, and B. Ramabhad-ran, "Deep convolutional neural networks for LVCSR," in Proc. of ICASSP , 2013.

[23] C. Zhang, K. Koishida, and J. Hansen, "Text-independent speaker verification based on triplet convolutional neural network embedding," IEEE Transactions on Audio, Signal, and Language Processing, vol. 26, no. 9, pp. 1633-1644, 2018.

[24] A. Nagrani, J. S. Chung, and A. Zisserman, "Voxceleb:a large scale speaker identification dataset," in Proc. of Interspeech, 2017.

[25] D. Palaz, M. Magimai-Doss, and R. Collobert, "Analysis of CNNbased speech recognition system using raw speech as input," in Proc. of Interspeech , 2015.

[26] H. Muckenhirn, M. Magimai-Doss, and S. Marcel, "To-wards directly modeling raw speech signal for speaker verification using CNNs," in Proc. of ICASSP , 2018.

[27] Joakim Andèn and Stephane Mallat, "Deep scattering spectrum," IEEE Transactions on Signal Processing, vol. 62, no. 16, pp. 4114-4128, 2014.

[28] E. Oyallon and S. Mallat, "Deep roto-translation scattering for object classification," in Proc. of IEEE Conference on Computer Vision and Pattern Recognition , 2015, pp. 2865-2873.

[29] Stephane Mallat, "Group invariant scattering," Communications on Pure and Applied Mathematics , vol. 65, no. 10, pp. 1331-1398, 2012.

[30] Vaclav Chuda cek, Joakim And en, Stephane Mallat, Patrice Abry, and Muriel Doret, "Scattering transform for intra-partum fetal heart rate variability fractal analysis: A case-control study," IEEE Transactions on Biomedical Engineering vol. 61, no. 4, pp. 1100-1108, 2014.

[31] V. Peddinti, T. N. Sainath, S. Maymon, B. Ramabhadran, D. Nahamoo, and V. Goel, "Deep scattering spectrum with deep neural network," in Proc. of ICASSP , 2014, pp. 361-364.

[32] P. Fousek, P. Dognin, and V. Goel, "Evaluating deep scattering spectra with deep neural networks on large-scale spontaneous speech task," in Proc. of ICASSP, 2015, p. 54.

[33] L. Lamel, and R. Kassel, and S. Seneff, "Speech Database Development: Design and Analysis of the Acoustic-Phonetic Corpus," Proc. of DARPA Speech Recognition Work-shop, 1986. 
[34] V. Panayotov, G. Chen, D. Povey, and S. Khudanpur, "Librispeech: An ASR corpus based on public domain audio books," Proc. of ICASSP, pp. 5206-5210, 2015

[35] M. Ravanelli and Y. Bengio, "Speaker Recognition from raw waveform with SincNet," Proc. of SLT, 2018.

[36] H. Muckenhirn, M. Magimai-Doss, and S. Marcel, "On Learning Vocal Tract System Related Speaker Discriminative Information from Raw Signal Using CNNs,” Proc. of Interspeech, 2018. 